\title{
Influência da pedalada com os joelhos tangenciando o quadro da bicicleta sobre a ativação dos músculos do membro inferior
}

CDD. 20.ed. 616.7

796.6

\section{Resumo}

A mudança da posição do corpo sobre a bicicleta tem sido relacionada a alterações na ativação dos músculos do membro inferior. Desta forma, o objetivo do presente estudo foi comparar a ativação dos músculos "Tibialis Anterior", "Gastrocnemius Medialis", "Biceps Femoris", "Rectus Femoris", "Vastus Lateralis", "Adductor Longus" e "Gluteus Maximus" nas seguintes situações: 1) posição de referência (posição preferida); 2) posição de adução (joelhos tangenciando o quadro da bicicleta); 3) posição de abdução (joelhos afastados do quadro da bicicleta). Seis atletas com experiência competitiva em ciclismo foram avaliados por meio da eletromiografia de superfície (EMG). Todos pedalaram em suas próprias bicicletas montadas em um ciclosimulador, com carga de trabalho normalizada pelo $\mathrm{VO}_{2}$ de forma que a taxa de troca respiratória se mantivesse entre 0,8 e 1,0. A ativação muscular foi analisada por meio da comparação da média do envelope RMS e do período de ativação para cada um dos músculos, nas três posições avaliadas. Não foram observadas diferenças significativas para a média do envelope RMS e para o período de ativação dos músculos nas três posições avaliadas, à exceção do "Adductor Longus". Observou-se maior ativação $(36 \pm 6 \%)$ deste músculo na posição de adução comparado a posição de abdução $(25 \pm 11 \%)$ para um valor de significância de $p=0,02$, sem diferenças em relação a posição de referência $(27 \pm 7 \%)$. Estes resultados sugerem que não ocorrem alterações substanciais na ativação dos principais músculos do membro inferior quando a posição dos joelhos no plano frontal é alterada e a carga de trabalho é mantida, à exceção do aumento da participação do "Adductor Longus".

UnIteRMos: Ciclismo; Eletromiografia; Aerodinâmica.

\section{Introdução}

A otimização dos aspectos aerodinâmicos relacionados à postura do ciclista sobre a bicicleta desempenha um importante papel para a melhora do desempenho (McCole, Claney, Conte, Anderson \& HagberG, 1990). Uma estratégia em relação às características aerodinâmicas comumente empregada pelos ciclistas em competições é pedalar em uma posição com os joelhos tangenciando o quadro da bicicleta por meio da adução dos quadris. Ciclistas assumem tal posição com o objetivo primário de reduzir a área frontal do conjunto ciclista-bicicleta (BURKE \& PRUTTT, 2003).

Resultados prévios (BIni, Carpes \& DiefEnTHAELER, 2009) indicam que é possível aumentar a força resultante quando pedalando com os joelhos tangenciando o quadro da bicicleta (posição de adução) comparado com a posição dos joelhos preferida pelo atleta em relação ao quadro da bicicleta (posição de referência). Uma das possíveis razões para explicar essas observações seria um aumento no comprimento dos músculos extensores mono-articulares do quadril (i.e. "Gluteus Maximus"), o que levaria estes músculos a trabalhar em uma região mais produtiva da relação força-comprimento muscular. A adaptação dos músculos adutores do quadril para a produção de força em menores comprimentos musculares devido à tentativa, por parte do ciclista, em manter os joelhos próximos ao quadro da bicicleta durante a pedalada, também seria uma possível explicação. Esta tentativa foi descrita por BURKe e PRUITT 
(2003), como sendo frequentemente utilizada em competições de ciclismo, principalmente durante as etapas de contra-relógio individual.

A posição do corpo tem significante influência sobre a ativação dos músculos do membro inferior. A mudança no ângulo de flexão do tronco, por exemplo, provoca alteraçóes na ativação de todos os músculos do membro inferior avaliados, incluindo músculos que não cruzam a articulação do quadril (ex. "Tibialis Anterior" e "Soleus") (SAVELbERG, VAN De Port \& Willems, 2003). Dentre as possíveis razões para estas mudanças na ativação muscular do membro inferior, salienta-se a alteração do ângulo do tronco, o que relacionado com a diferente função dos músculos mono e bi-articulares (HOF, 2001; ZAJAC, 2002), ou mesmo a influência do comprimento muscular (YoshinUKu \& HeRzoG, 1996), leva a mudanças globais no padrão de recrutamento muscular (Diefenthaeler, Bini, Karolczak \& Carpes, 2008).

O padrão de ativação dos músculos do membro inferior no ciclismo é descrito na literatura (BAUM \& Li, 2003; DiefenthAeler et al., 2008). No entanto, os músculos adutores permaneceram em segundo plano nestas análises. HoutZ e FiSHER (1959) descreveram qualitativamente a ativação do músculo "Gracilis" no ciclismo, enquanto Watanabe, Katayama, Ishida e AKIMA (2009) avaliaram a ativação dos músculos
"Adductor Longus" e "Adductor Magnus" durante exercício de carga progressiva no ciclismo. Estes observaram ativação do "Gracilis" na região próxima aos 180 graus do ciclo da pedalada, provavelmente atuando como flexor do joelho, enquanto os músculos "Adductor Longus" e "Adductor Magnus" foram recrutados durante todo o ciclo de pedalada. Com isto, percebe-se uma carência de estudos com objetivo de investigar o efeito da pedalada com os joelhos tangenciando o quadro da bicicleta sobre a atividade dos músculos do membro inferior. Dessa forma, medir a ativação muscular nessa posição permitiria estimar a contribuição dos músculos adutores do quadril em relação aos demais músculos produtores de movimento durante o ciclismo.

Sendo assim, o objetivo do presente estudo foi comparar a ativação de músculos do membro inferior durante a pedalada, quando os ciclistas tangenciavam com os joelhos o quadro da bicicleta, em relação à posição preferida dos joelhos em relação ao quadro da bicicleta era adotada, e quando os ciclistas assumiram uma postura de abdução dos quadris, na qual os joelhos se mantinham afastados do quadro da bicicleta. Como hipótese secundária, estas alterações na ativação se estenderiam aos demais músculos do membro inferior mesmo nas posições diferentes daquela preferida pelo atleta.

\section{Material e métodos}

\section{Sujeitos}

Seis atletas com experiência competitiva no ciclismo (três ciclistas e três triatletas) participaram voluntariamente do estudo. O presente estudo foi aprovado pelo Comitê de Ética em Pesquisa com

Seres Humanos da Universidade onde o estudo foi realizado. Na TABELA 1 são apresentados os resultados referentes às características dos atletas avaliados, incluindo idade, tempo de treinamento (experiência) e volume semanal de treinamento de ciclismo.

TABELA 1 - Características dos atletas avaliados, incluindo idade, tempo de treinamento em anos, e distância semanal de treinamento de ciclismo em quilômetros (DP, desvio-padrão).

\begin{tabular}{lccc}
\hline \multicolumn{1}{c}{ Atleta } & Idade (anos) & Tempo de treino (anos) & Volume semanal $(\mathbf{k m})$ \\
\hline Ciclista A & 30 & 18 & 450 \\
Ciclista B & 24 & 4 & 600 \\
Ciclista C & 26 & 7 & 450 \\
Triatleta A & 43 & 3 & 200 \\
Triatleta B & 21 & 4 & 230 \\
Triatleta C & 23 & 1 & 200 \\
Média & 28 & 6 & 355 \\
DP & 8 & 6 & 168 \\
\hline
\end{tabular}




\section{Protocolo}

Os atletas foram avaliados em suas próprias bicicletas montadas em um ciclosimulador Cateye CS1000 (Cateye CO, Osaka, Japão). Com o intuito de auxiliar os atletas na manutenção das posturas avaliadas foram utilizadas hastes metálicas posicionadas lateralmente aos atletas, no sentido ânteroposterior, em paralelo a bicicleta, auxiliando na manutenção do afastamento dos joelhos do quadro da bicicleta, resultante da abdução do quadril.

Três posiçôes dos joelhos foram avaliadas: 1) Posição de Referência - REF: aquela em que o atleta pedalava com os joelhos na distância preferida do quadro da bicicleta; 2) Posição de Adução - ADU: aquela na qual o atleta pedalava com os joelhos tangenciando o quadro da bicicleta; 3) Posição de Abdução - ABDU: aquela quando o atleta pedalava com os joelhos afastados do quadro da bicicleta, quase tocando as hastes metálicas descritas anteriormente. Esta posição era mantida por meio de uma abdução dos quadris. $\mathrm{Na}$ FIGURA 1, são apresentadas as posições de Adução e Abdução, no plano frontal de um atleta.

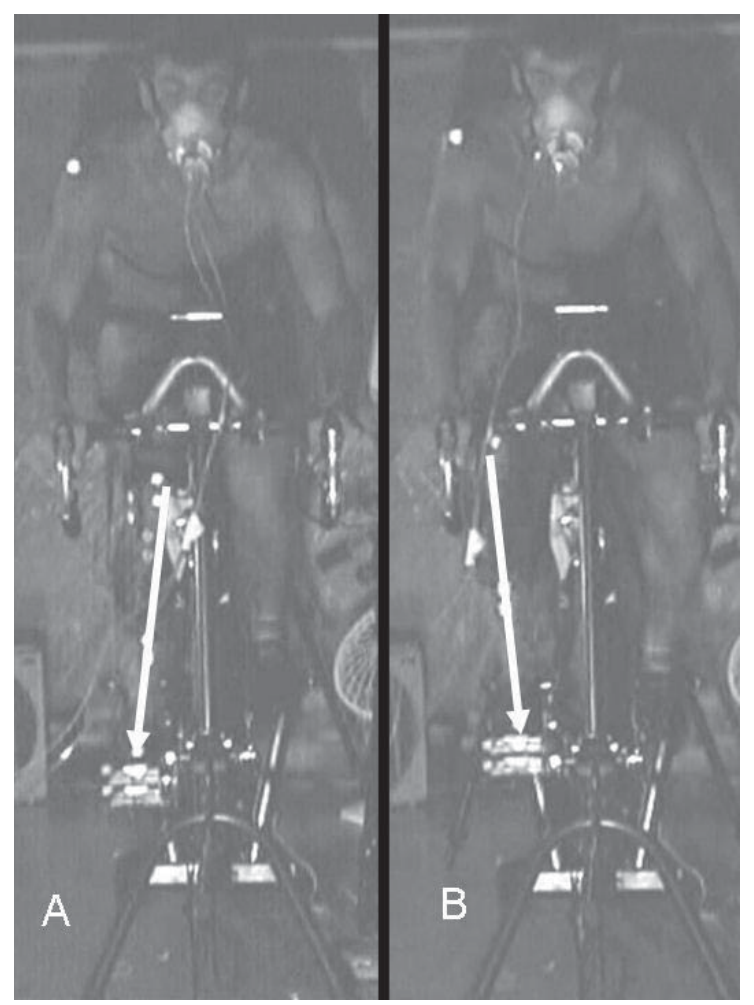

FIGURA 1 - Ilustração das posições de Adução (A) e Abdução (B). Na figura é representado o vetor queilustra a orientação da aplicação de força estimada no plano frontal em cada uma das posições por meio da análise qualitativa do alinhamento do segmento perna do ciclista.
Foram mensurados o consumo de oxigênio $\left(\mathrm{VO}_{2}\right)$, a produção de dióxido de carbono $\left(\mathrm{VCO}_{2}\right)$ e a taxa de troca respiratória (RER) por meio de um sistema de espirometria de circuito aberto CPX/D (Medical Graphics Corp., St Louis, USA) com coleta dos gases a cada respiração.

A carga de trabalho foi normalizada, para as três posições avaliadas, por meio da manutenção do $\mathrm{VO}_{2}$ no qual o RER se mantivesse com valores entre $0,8 \mathrm{e}$ 1,0 , indicando que os atletas estavam se exercitando em intensidade de esforço próxima àquela correspondente ao segundo limiar ventilatório (AMANN, Subudhi \& Foster, 2004; Solberg, Robstad, SKJønSBERG \& BORCHSENIUS, 2005). Estas condiçōes foram mantidas por meio da regulagem da inclinação do ciclosimulador em 3\%, relação de marchas e cadência fixas nas três posições avaliadas, sendo estas selecionadas durante o período de aquecimento.

\section{Coleta dos dados}

Nas condições previamente descritas, os atletas pedalaram por três minutos com o $\mathrm{VO}_{2}$ e o RER estabilizados. Os dados foram coletados nos últimos 30 segundos, sendo neste período adquiridos os dados de eletromiografia (EMG), eletrogoniometria e as variáveis respiratórias ( $\mathrm{VO}_{2}, \mathrm{VCO}_{2}$ e RER).

O cálculo da cadência média de pedalada em cada uma das posiçôes foi realizado por meio da análise do sinal advindo de um "reed switch" acoplado ao quadro da bicicleta. Este, compondo o sistema de eletrogoniometria, emitia um pulso elétrico a cada vez que o pé-de-vela passava por ele, caracterizando cada ciclo de pedalada (Diefenthaeler et al., 2008; Hug, Decherchi, Marqueste \& Jammes, 2004).

A atividade elétrica dos músculos "Tibialis Anterior", "Gastrocnemius Medialis", cabeça longa do "Bíceps Femoris", "Rectus Femoris", "Vastus Lateralis", "Adductor Longus" e "Gluteus Maximus" do membro inferior direito foi mensurada por meio da eletromiografia de superfície. Eletrodos Ag/ $\mathrm{AgCl}$ na configuração bipolar $(2,5 \mathrm{~cm}$ de distância entre eletrodos) foram posicionados sobre a pele, na região do ventre muscular após tricotomia e limpeza da pele com abrasão a base de álcool, respeitando as recomendações para colocação dos eletrodos (Merletti, Botter, Troiano, Merlo \& MinetTo, 2009). Um eletrodo de referência foi posicionado sobre a superfície anterior da tibia. Os cabos do sistema de EMG foram fixados a pele, permitindo a movimentação do segmento inferior do atleta e minimizando o movimento dos fios 
durante a coleta dos dados. Os sinais de EMG foram adquiridos pelo "software" Windaq ${ }^{\circledR}$ (WINDAQ, DataQ Instruments Inc., USA) com uma taxa de amostragem de $2000 \mathrm{~Hz}$ por canal, e amplificação de $1 \mathrm{~K}$, utilizando-se um eletromiógrafo de oito canais (Bortec Eletronics Inc., Calgary, Canadá).

\section{Análise dos dados}

Os dados de EMG foram submetidos a um filtro digital do tipo Butterworth de quinta ordem do tipo passa-banda, com frequência de corte de $10-500 \mathrm{~Hz}$ (Merletti et al., 2009). Da mesma forma, os dados de eletrogoniometria foram analisados em rotinas desenvolvidas em ambiente MATLAB® (Math Works Inc., EUA). Nestas rotinas foi calculado o envelope RMS do sinal de EMG, com janela retangular de 0,04 segundos (NePtune, KaUtz \& Hull, 1997), para subsequente análise da média dos 10 primeiros ciclos alternados de pedalada. O envelope RMS foi normalizado pelo maior valor obtido na posição de referência (Bini, CARPES, DiefentHAeler, Mota \& Guimarâes, 2008). O período de ativação foi determinado como o percentual do ciclo da pedalada no qual o músculo atingisse ativação maior do que $10 \%$ do seu valor máximo (BAUM \& Li, 2003; Diefenthaeler et al., 2008), como representado na FIGURA 2.

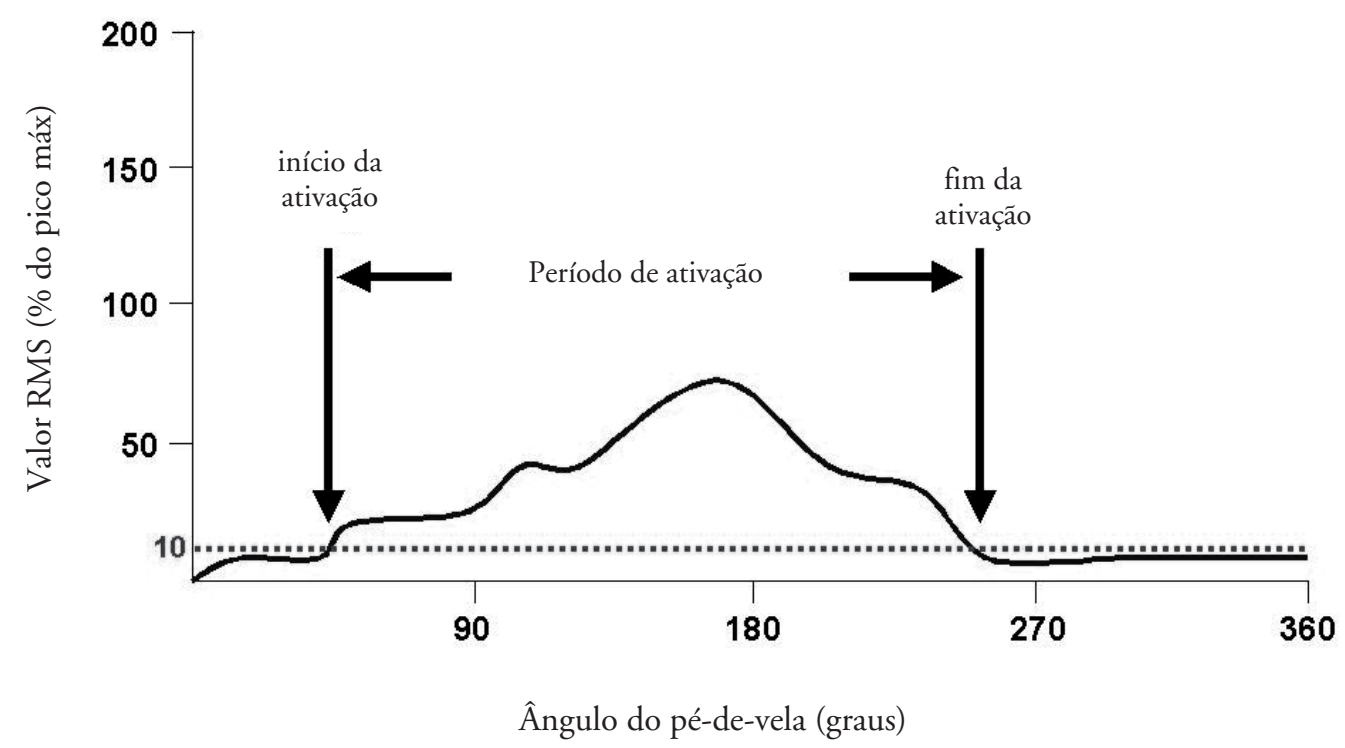

FIGURA 2 - Representação do critério utilizado para determinar o período de ativação muscular (DIEFENTHAELER et al., 2008).

Os dados foram inicialmente analisados por meio de estatística descritiva utilizando a média e o desvio-padrão dos seis atletas avaliados. Para a análise estatística foi utilizada uma análise de variância para medidas repetidas de um fator (ANOVA), com o objetivo de comparar as variâncias do valor médio do envelope RMS e do período de ativação de cada um dos 10 ciclos de pedalada, assim como da cadência de pedalada entre as posiçõos de referência, adução e abdução. Este foi seguido pelo teste "post-hoc" HSD de Tukey quando diferenças significativas fossem observadas. Para todos os procedimentos estatísticos utilizou-se o pacote estatístico SigmaStat 2.03 (SPSS Inc., EUA) com nível de significância de 0,05. 


\section{Resultados}

A cadência de pedalada não foi alterada significativamente com a mudança na posição dos joelhos em relação ao quadro da bicicleta (REF $88 \pm 7 \mathrm{rpm}$; ADU $94 \pm 10 \mathrm{rpm} ; \mathrm{ABDU} 95 \pm 9 \mathrm{rpm}$, para $\mathrm{p}=0,06)$.
Na FIGURA 3 é apresentado o comportamento da ativação dos músculos avaliados do ciclista $\mathrm{A}$, representativo do grupo avaliado, na posição de referência.
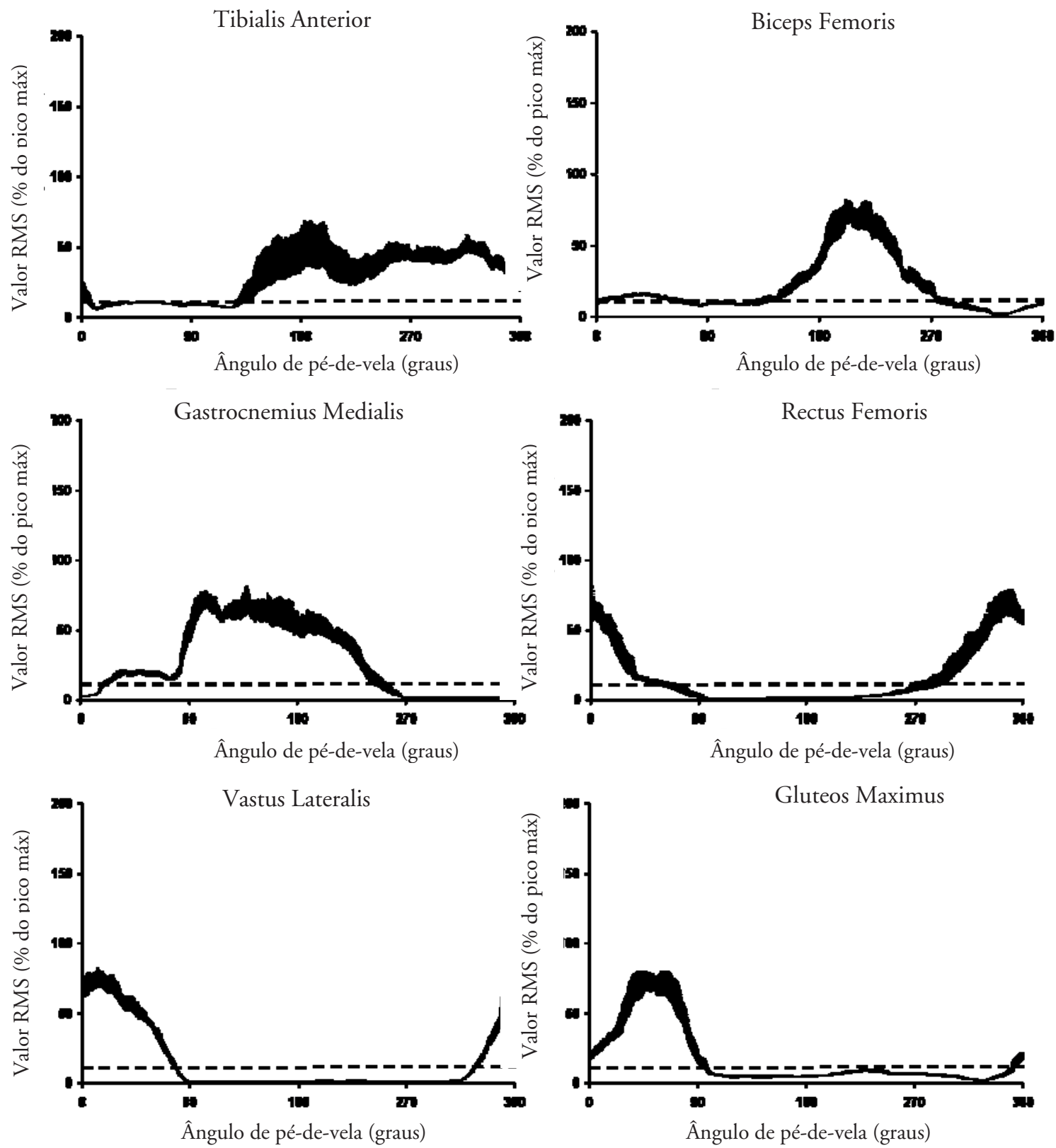

FIGURA 3 - Representação da curva média (+DP) de 10 ciclos de pedalada do RMS dos músculos avaliados do ciclista A pedalando na posição de referência.

O padrão de ativação observado no presente estudo apresenta-se de acordo com resultados prévios descritos na literatura (BAUM \& LI, 2003; Bini et al., 2008).
Na FIGURA 4 é apresentado o padrão de ativação do músculo "Adductor Longus" do ciclista A, representativo do grupo avaliado, nas posições de referência, adução e abdução.
A linha tracejada indica $10 \%$ do pico máximo de ativação, limiar adotado como critério de ativação. 
O padrão de ativação é apresentado em relação à curva média e desviopadrão do RMS nos 10 ciclos de pedalada. A linha tracejada indica $10 \%$ do pico máximo de ativação, limiar adotado como critério de ativação.
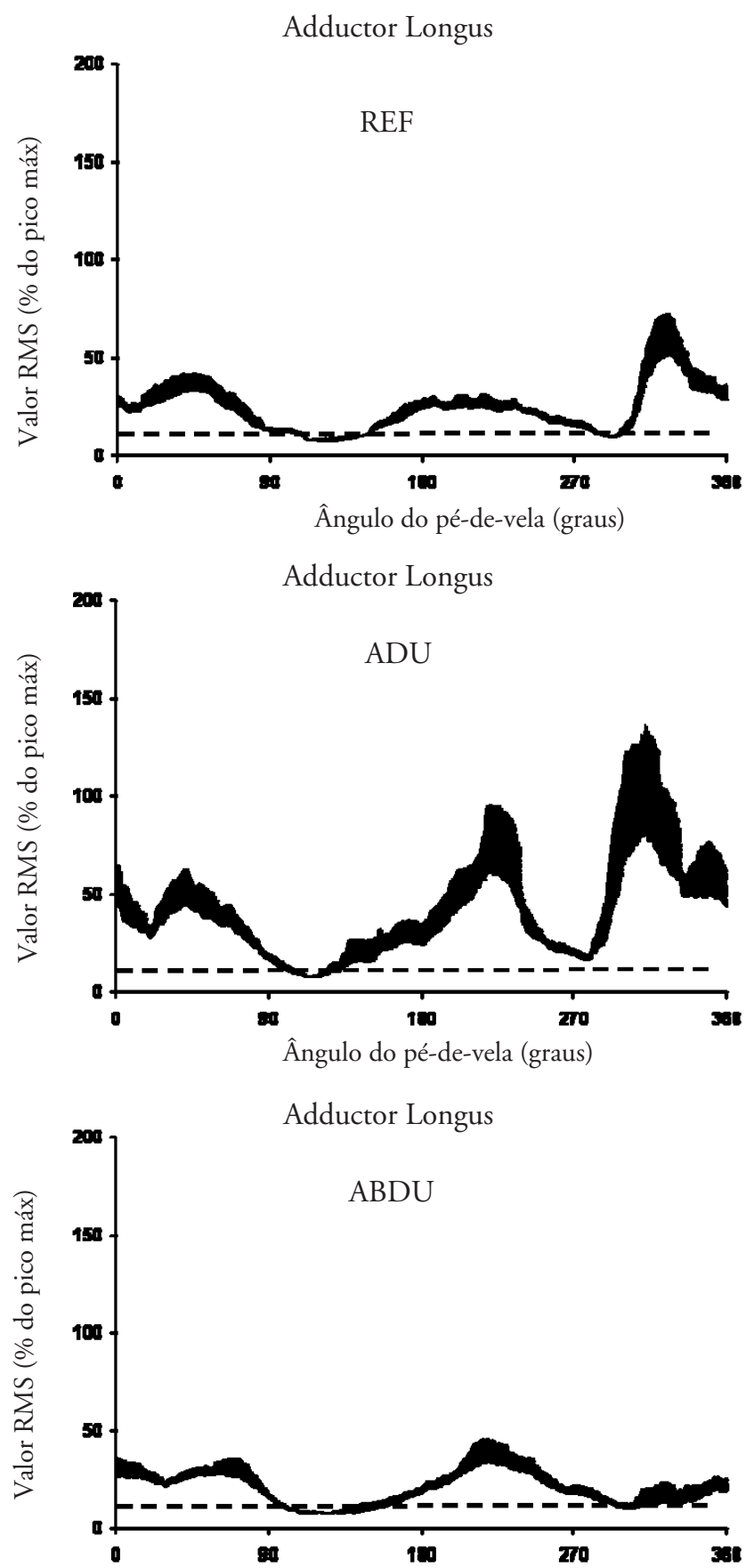

Ângulo do pé-de-vela (graus)

FIGURA 4 - Representação do RMS do músculo “Adductor Longus” do ciclista A nas posições dereferência (REF), adução (ADU) e abdução (ABDU).

Na FIGURA 5 são apresentados os resultados da média e desvio-padrão (DP) do valor médio do envelope RMS dos seis sujeitos avaliados. Os resultados são apresentados para os sete músculos analisados, nas três posições avaliadas.

Os resultados indicam redução significativa do envelope RMS $(\mathrm{P}<0,05)$ na média dos 10 ciclos de pedalada na posição de abdução quando comparada à posição de adução, para o músculo "Adductor Longus". Ambas as posiçôes não diferiram da posição de referência para este músculo. Os demais músculos não apresentaram diferenças estatisticamente significativas entre as posições avaliadas.

Na FIGURA 6 são apresentados os resultados da média e desvio-padrão (DP) do período de ativação dos seis sujeitos avaliados. Os resultados são apresentados para os sete músculos analisados, nas três posiçōes avaliadas.Os resultados do período de ativação indicaram que não houve diferença significativa entre as posições do joelho avaliadas no presente estudo. 


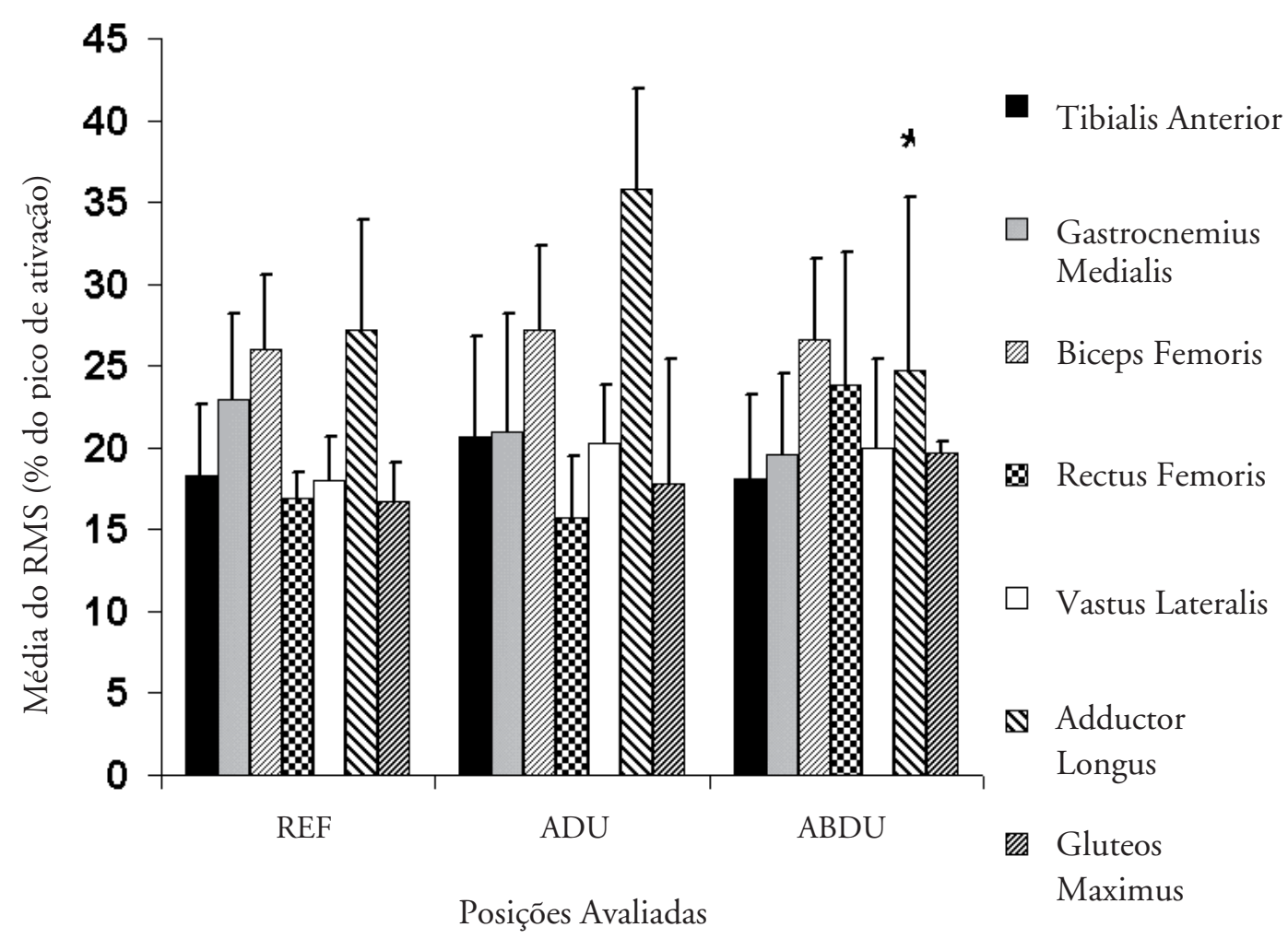

FIGURA 5 - Média e DP do RMS dos músculos “Tibialis Anterior”, "Gastrocnemius Medialis”, "Biceps Femoris”, "Rectus Femoris", "Vastus Lateralis", "Adductor Longus" e "Gluteus Maximus" nas posições de referência (REF), adução (ADU) e abdução (ABDU).

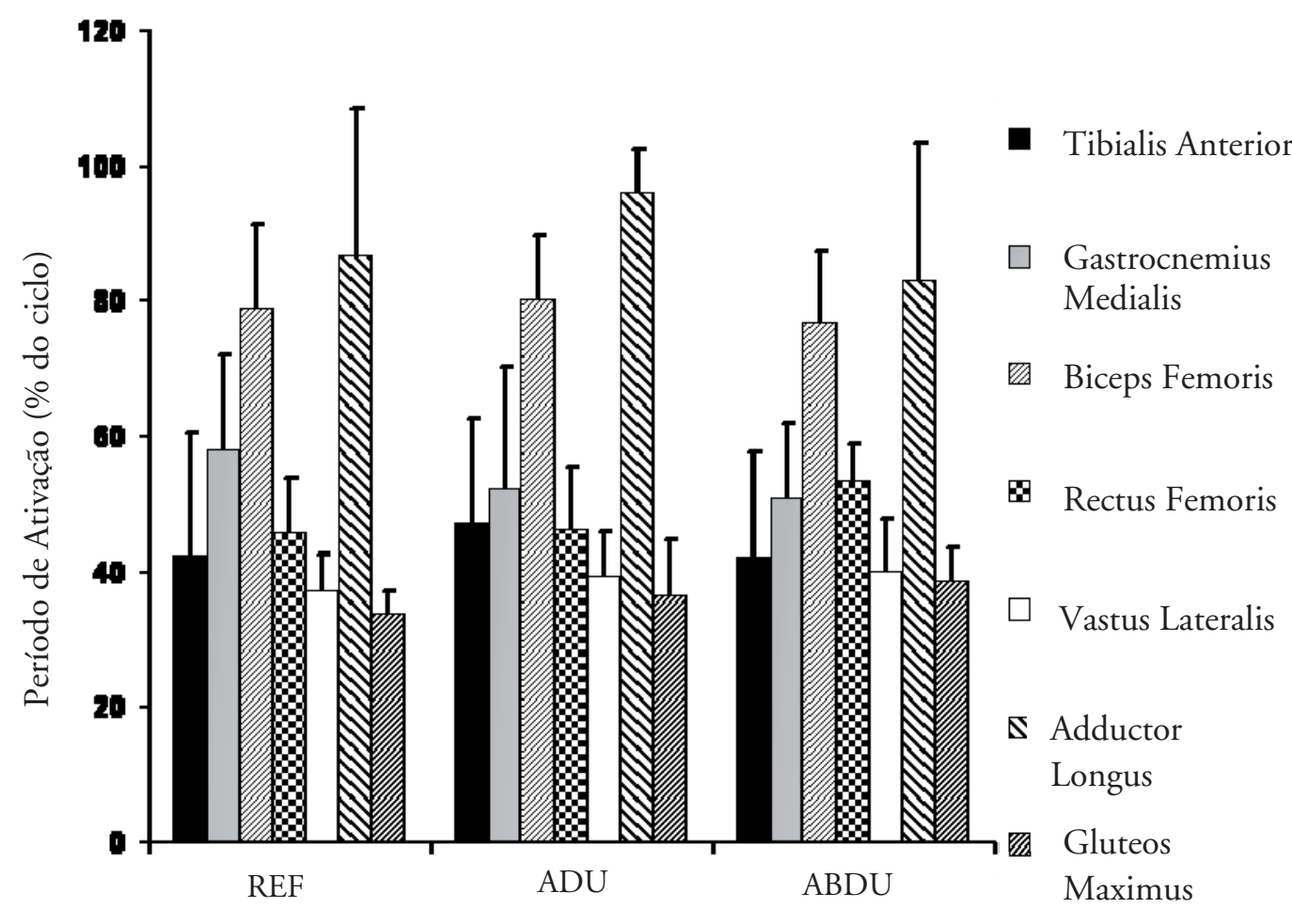

Posições Avaliadas

FIGURA 6 - Média e DP do período de ativação dos músculos “Tibialis Anterior", "Gastrocnêmius Medialis", "Bíceps Femoris", "Rectus Femoris", "Vastus Lateralis", "Adductor Longus" e "Gluteus Maximus" nas posições de referência (REF), adução (ADU) e abdução (ABDU). 


\section{Discussão}

Este trabalho teve como objetivo comparar a ativação dos músculos do membro inferior durante a pedalada tangenciando os joelhos ao quadro da bicicleta em relação à posição preferida do atleta e a posição de abdução dos quadris. A hipótese inicial era de que ao tangenciar o quadro da bicicleta com os joelhos, ocorreria um aumento na ativação dos músculos adutores do quadril. Esperava-se também que ocorresse mudança na ativação dos demais músculos do membro inferior em resposta a mudança na postura do ciclista. Os resultados encontrados confirmaram parcialmente as hipóteses, visto que apenas o músculo "Adductor Longus" apresentou alterações no RMS quando foi alterada a posição dos joelhos em relação ao quadro da bicicleta.

DiefentHAELER et al. (2008) observaram que com alterações na posição do selim de ciclistas ocorrem mudanças na magnitude e no período de ativação dos músculos do membro inferior. No entanto, o efeito da mudança na posição do selim não resultou em mudanças no período de ativação dos principais músculos do membro inferior, como observado no presente estudo para as mudanças na posição dos joelhos. Chapman, Vicenzino, Blanch e Hodges (2008) indicaram que alterações na mecânica da pedalada como aquelas observadas quando é alterada a cadência de pedalada (ex. alteração na velocidade de contração dos músculos), resultam em mudanças na amplitude da ativação, mas não no período de ativação. Estes autores justificam este aspecto por meio da proposição de que o gesto da pedalada seria um padrão motor consolidado por meio do treinamento.

Savelberg, Van de Port e Willems (2003) e Dorel, Couturier e Hug (2009) observaram que a mudança no ângulo do tronco exigiu "compensações" do ponto de vista da ativação muscular para a manutenção da carga de trabalho. Estas "compensações" dizem respeito a mudanças na magnitude de ativação dos músculos do membro inferior para que fosse mantida a força produzida. Hug et al. (2004) observaram que o aumento da carga de trabalho é compensado pelo aumento da ativação do músculo "Vastus Lateralis". No entanto, a análise do $\mathrm{VO}_{2} \mathrm{e}$ da potência produzida indicaram que a demanda fisiológica e a carga de trabalho parecem não ser alteradas na posição de adução em relação à posição de referência, como demonstrou estudo anterior (Bini, Diefenthaeler, Carpes, Nabinger, Mota \& GUIMARÃES, 2006). Este resultado corrobora com a ausência de diferenças significativas no RMS dos demais músculos do membro inferior para a mesma carga de trabalho.

A similaridade do RMS nas posições de referência e adução sugere uma adaptação muscular destes atletas. Sabe-se que o treinamento gera uma adaptação muscular ao comprimento no qual o músculo é exigido (Herzog, Guimaraes, Anton \& Carter-Erdman, 1991; SAVELBERg \& MeIJeR, 2003), ou mesmo a uma faixa de comprimento muscular próxima ao comprimento muscular treinado (KITAI \& Sale, 1989). Sabendo-se que a estratégia de tangenciar com os joelhos o quadro da bicicleta é mais utilizada pelos atletas em treinamentos e competições do que a posição de abdução (BURKE \& PRUITT, 2003), pode-se inferir que pela utilização destes músculos (i.e. "Adductor Longus") nestas posições (referência e adução), ocorreria uma adaptação da musculatura a estas posições. A avaliação do posicionamento dos joelhos no plano frontal nestas posiçôes poderia indicar a magnitude da diferença na posição do membro inferior entre as condiçōes avaliadas no presente estudo.

O ciclismo, sendo um movimento realizado em cadeia cinética fechada, acaba por ativar um número importante de músculos, exigindo inúmeras estratégias de ativação destes músculos (HoF, 2001; ZAJAC, 2002). Esta análise poderia indicar que não apenas os sete músculos avaliados no presente estudo poderiam ser utilizados de forma aumentada para a maior aplicação de força no pedal, como observado quando realizada a adução dos quadris durante a pedalada. Esta redundância motora justificaria a similaridade do RMS dos músculos analisados nas três posições avaliadas.

O padrão de ativação dos músculos adutores do quadril foi pouco abordado na literatura até o presente momento. Houtz e FisCHER (1959) indicaram que o músculo "Gracilis" parece atuar como estabilizador da posição do joelho próximo ao quadro da bicicleta, assim como flexor de joelho. WatanaBE et al. (2009) descreveram a ativação dos músculos "Adductor Longus" e "Adductor Magnus" como ocorrendo durante todo o ciclo da pedalada. No presente estudo, a escolha pelo monitoramento do músculo "Adductor Longus" permitiu analisar um músculo representativo do grupo dos adutores do quadril mono-articulares, contrário ao realizado por Houtz e FisCher (1959). Desta forma observouse, qualitativamente, que o músculo "Adductor Longus" parece ser responsável pela manutenção do joelho próximo ao quadro da bicicleta. GREGOR 
(2000) analisou resultados do comportamento do joelho no plano frontal ao longo do ciclo de pedalada e indicou que durante a fase de propulsão ocorre aproximação dos joelhos do quadro da bicicleta, enquanto que após aproximadamente $270^{\circ}$ do ciclo do pé-de-vela este se afasta lateralmente do quadro da bicicleta. No presente estudo, foi observada a ativação do músculo "Adductor Longus" em três instantes do ciclo da pedalada, sendo dois destes na fase de recuperação (de 180 a $360^{\circ}$ do pé-de-vela). WatANABE et al. (2009) indicam que o músculo "Adductor Longus" pode auxiliar na flexão do quadril, o que explicaria a ativação aumentada deste após os $270^{\circ}$ do pé-de-vela. $\mathrm{O}$ monitoramento da cinemática do joelho nos planos frontal e sagital permitiria um melhor entendimento da função dos músculos adutores do quadril como "estabilizadores" da posição dos joelhos em relação ao quadro da bicicleta, assim como a sua possível atuação como flexor da articulação do quadril.

$\mathrm{O}$ presente estudo permitiu identificar que a alteração da posição do joelho no plano frontal não afeta de forma significativa os principais músculos relacionados com a produção de torque no ciclismo. No entanto, os músculos adutores foram significativamente mais ativados, o que pode aumentar a fatigabilidade destes músculos e interferir no desempenho do ciclista.

\title{
Conclusão
}

A estratégia de pedalar com os joelhos tangenciando o quadro da bicicleta não alterou a magnitute, tampouco o período de ativação, de sete músculos do membro inferior. Apenas o músculo "Adductor Longus" teve sua ativação diminuida na posição de abdução comparada às demais posições do joelho.

\begin{abstract}
Effects of cycling with the knees close to the bicycle frame on the lower limb muscle activation

Cyclist's body position on the bike has been related to changes in muscle activation. Therefore, the aim of the present study was to compare the activation of Tibialis Anterior, Gastrocnemius Medialis, Biceps Femoris, Rectus Femoris, Vastus Lateralis, Adductor Longus e Gluteus Maximus muscles in three conditions: 1) reference position (preferred position); 2) adduction position (knees almost touching the bicycle frame); 3) abduction position (knees away from the bicycle frame). Six athletes with competitive experience in cycling were evaluated using surface electromyography. They pedalled using their own bicycles mounted on a wind-trainer, with a workload relative to the second ventilatory threshold. Muscle activation was defined by the mean value of the RMS envelope and by the activation period for the three evaluated positions. There were no significant differences in muscles' RMS or activation period between the three positions, except for the Adductor Longus muscle. In adduction position, this muscle was more activated $(36 \pm 6 \%)$, compared to the abduction position $(25 \pm 11 \%)$ for a significance level of $p=0.02$, without significant differences for the reference position $(27 \pm 7 \%)$. These results indicate that there is no substantial difference in the activation of the most important muscles of the lower limb when the position of the knee is changed in the frontal plane for the same workload level, with the Adductor Longus as an exception.
\end{abstract}

UnITERMS: Cycling; Electromyography; Aerodynamics. 


\section{Referências}

AMANN, M.; SUBUDHI, A.; FOSTER, C. Influence of testing protocol on ventilatory thresholds and cycling performance. Medicine and Science in Sports and Exercise, Madison, v.36, n.4, p.613-22, 2004.

BAUM, B.S.; LI, L. Lower extremity muscle activities during cycling are influenced by load and frequency. Journal of Electromyography and Kinesiology, New York, v.13, n.2, p.181-90, 2003.

BINI, R.R.; CARPES, F.P.; DIEFENTHAELER, F. Efeitos da posição dos joelhos no plano frontal sobre as forças aplicadas no ciclismo: estudo preliminar. Revista Brasileira de Cineantropometria e Desempenho Humano, Florianopólis, v.11, n.2, p.142-50, 2009.

BINI, R.R.; CARPES, F.P.; DIEFENTHAELER, F.; MOTA, C.B.; GUIMARÃES, A.C.S. Physiological and electromyographic responses during 40-km cycling time trial: Relationship to muscle coordination and performance. Journal of Science and Medicine in Sport, Belconnen, v.11, n.4, p.363-70, 2008.

BINI, R.R.; DIEFENTHAELER, F.; CARPES, F.P.; NABINGER, N.; MOTA, C.B.; GUIMARĀES, A.C.S. Economia de movimento e pico de força no pedal durante o ciclismo em posição aerodinâmica. Revista Brasileira de Ciência e Movimento, São Caetano do Sul, v.14, p.288. 2006. Suplemento.

BURKE, E.R.; PRUITT, A.L. Body positioning for cycling. In: BURKE, R. (Ed.). High-tech cycling. Champaign: Human Kinetics, 2003. p.69-92.

CHAPMAN, A.; VICENZINO, B.; BLANCH, P.; HODGES, P.W. Patterns of leg muscle recruitment vary between novice and highly trained cyclists. Journal of Electromyography and Kinesiology, New York, v.18, n.3, p.359-71, 2008. DIEFENTHAELER, F.; BINI, R.R.; KAROLCZAK, A.P.; CARPES, F.P. Ativação muscular durante a pedalada em diferentes posições do selim. Revista Brasileira de Cineantropometria e Desempenho Humano, Florianopólis, v.10, n.2, p.161-9, 2008.

DOREL, S.; COUTURIER, A.; HUG, F. Influence of different racing positions on mechanical and electromyographic patterns during pedalling. Scandinavian Journal of Medicine and Science in Sports, Copenhagen, v.19, n.1, p.44-54. 2009. GREGOR, R.J. Biomechanics of cycling. In: GARRET, W.E; KIRKENDALL, D.T. (Eds.). Exercise and sports science. Philadelphia: Lippincott Williams \& Wilkings, 2000. p.549-71.

HERZOG, W.; GUIMARAES, A.C.; ANTON, M.G.; CARTER-ERDMAN, K.A. Moment-length relations of rectus femoris muscles of speed skaters/cyclists and runners. Medicine and Science in Sports and Exercise, Madison, v.23, n.11, p.1289-96, 1991.

HOF, A.L. The force resulting from the action of mono- and biarticular muscles in a limb. Journal of Biomechanics, New York, v.34, n.8, p.1085-9, 2001.

HOUTZ, S.J.; FISCHER, F.J. An analysis of muscle action and joint excursion during exercise on a stationary bicycle. Journal of Bone Joint and Surgery, Boston, v.41A, n.1, p.123-31, 1959.

HUG, F.; DECHERCHI, P.; MARQUESTE, T.; JAMMES, Y. EMG versus oxygen uptake during cycling exercise in trained and untrained subjects. Journal of Electromyography and Kinesiology, New York, v.14, n.2, p.187-95, 2004. KITAI, T.A.; SALE, D.G. Specificity of joint angle in isometric training. European Journal of Applied Physiology and Occupational Physiology, Berlin, v.58, n.7, p.744-8, 1989.

McCOLE, S.D.; CLANEY, K.; CONTE, J.C.; ANDERSON, R.; HAGBERG, J.M. Energy expenditure during bicycling. Journal of Applied Physiology, Bethesda, v.68, n.2, p.748-53, 1990.

MERLETTI, R.; BOTTER, A.; TROIANO, A.; MERLO, E.; MINETTO, M.A. Technology and instrumentation for detection and conditioning of the surface electromyographic signal: state of the art. Clinical Biomechanics, Bristol, v.24, n.2, p.122-34, 2009.

NEPTUNE, R.R.; KAUTZ, S.A.; HULL, M.L. The effect of pedaling rate on coordination in cycling. Journal of Biomechanics, New York, v.30, n.10, p.1051-8, 1997.

SAVELBERG, H.H.C.M.; MEIJER, K. Contribution of mono- and biarticular muscles to extending knee joint moments in runners and cyclists. Journal of Applied Physiology, Bethesda, v.94, n.6, p.2241-8, 2003.

SAVELBERG, H.H.C.M.; VAN de PORT, I.G.L.; WILLEMS, P.J.B. Body configuration in cycling affects muscle recruitment and movement pattern. Journal of Applied Biomechanics, Champaign, v.19, n.4, p.310-24, 2003.

SOLBERG, G.; ROBSTAD, B.; SKJØNSBERG, O.H.; BORCHSENIUS, F. Respiratory gas exchange indices for estimating the anaerobic threshold. Journal of Sports Science and Medicine, Bursa, v.4, n.1, p.29-36, 2005.

WATANABE, K.; KATAYAMA, K.; ISHIDA, K.; AKIMA, H. Electromyographic analysis of hip adductor muscles during incremental fatiguing pedaling exercise. European Journal of Applied Physiology, Berlin, v.106, n.6, p.815-25, 2009. 
YOSHIHUKU, Y.; HERZOG, W. Maximal muscle power output in cycling: a modelling approach. Journal of Sports Sciences, London, v.14, n.2, p.139-57, 1996.

ZAJAC, F.E. Understanding muscle coordination of the human leg with dynamical simulations. Journal of Biomechanics, New York, v.35, n.8, p.1011-8, 2002.

\section{Agradecimentos}

Os autores gostariam de agradecer ao CNPq e CAPES pelo aporte financeiro para a realização deste projeto, assim como todos os que colaboraram com a execução deste projeto.

Dedicamos este trabalho ao professor Antônio Carlos Stringhini Guimarães que idealizou e participou ativamente deste projeto até o seu falecimento.

\begin{tabular}{r|r} 
ENDEREÇO & \\
Rodrigo Rico Bini & \\
Sport Performance Research Institute New Zealand & \\
School of Sport and Recreation & Recebido para publicação: 22/ 02/ 2010 \\
Auckland University of Technology & Revisado: 11/06/2010 \\
Private Bag 92006 & Aceito: 08/07/2010 \\
Auckland - New Zealand & \\
e-mail: bini.rodrigo@gmail.com & \\
\hline
\end{tabular}

